\title{
Measurement of the Mass of the $\tau$ Lepton
}

J. Z. Bai, ${ }^{(1)}$ O. Bardon, ${ }^{(6)}$ R. A. Becker-Szendy, ${ }^{(7)}$ T. H. Burnett, ${ }^{(10)}$ J. S. Campbell, ${ }^{(9)}$ S. J. Chen, ${ }^{(1)}$ S. M. Chen, (1) Y. Q. Chen, (1) Z. D. Cheng, ${ }^{(1)}$ J. A. Coller, ${ }^{(2)}$ R. F. Cowan, ${ }^{(6)}$ H. C. Cui, ${ }^{(1)}$ X. Z. Cui, ${ }^{(1)}$ H. L. Ding, ${ }^{(1)}$ Z. Z. Du, (1) W. Dunwoodie, ${ }^{(7)}$ C. Fang, ${ }^{(1)}$ M. J. Fero, ${ }^{(6)}$ M. L. Gao, ${ }^{(1)}$ S. Q. Gao, ${ }^{(1)}$ W. X. Gao, ${ }^{(1)}$ Y. N. Gao, ${ }^{(1)}$ J. H. Gu, (1) S. D. Gu, (1) W. X. Gu, ${ }^{(1)}$ Y. N. Guo, ${ }^{(1)}$ Y. Y. Guo, ${ }^{(1)}$ Y. Han, ${ }^{(1)}$ M. Hatanaka, (3) J. He, (1) D. G. Hitlin, ${ }^{(3)}$ G. Y. Hu, (1) T. Hu, (1) D. Q. Huang, ${ }^{(1)}$ Y. Z. Huang, (1) J. M. Izen, ${ }^{(9)}$ Q. P. Jia, (1) C. H. Jiang, ${ }^{(1)}$ Z. J. Jiang, ${ }^{(1)}$ A. S. Johnson, ${ }^{(2)}$ L. A. Jones, ${ }^{(3)}$ M. H. Kelsey, ${ }^{(3)}$ Y. F. Lai, ${ }^{(1)}$ P. F. Lang, ${ }^{(1)}$ A. Lankford, ${ }^{(4)}$ F. Li, (1) ${ }^{(1 . ~ L i, ~}{ }^{(1)}$ P. Q. Li, ${ }^{(1)}$ Q. M. Li, ${ }^{(1)}$ R. B. Li, ${ }^{(1)}$ W. Li, ${ }^{(1)}$ W. D. Li, (1) W. G. Li, ${ }^{(1)}$ Y. S. Li, (1) S. Z. Lin, ${ }^{(1)}$ H. M. Liu, ${ }^{(1)}$ Q. Liu, ${ }^{(1)}$ R. G. Liu, ${ }^{(1)}$ Y. Liu, ${ }^{(1)}$ B. Lowery, ${ }^{(9)}$ J. G. Lu, ${ }^{(1)}$ D. H. Ma, ${ }^{(1)}$ E. C. Ma, ${ }^{(1)}$ J. M. Ma, ${ }^{(1)}$ M. Mandelkern, ${ }^{(4)}$ H. Marsiske, ${ }^{(7)}$ H. S. Mao, (1) Z. P. Mao, ${ }^{(1)}$ X. C. Meng, ${ }^{(1)}$ H. L. Ni, ${ }^{(1)}$ L. J. Pan, ${ }^{(1)}$ J. H. Panetta, ${ }^{(3)}$ F. C. Porter, ${ }^{(3)}$ E. N. Prabhakar, ${ }^{(3)}$ N. D. Qi, (1) Y. K. Que, ${ }^{(1)}$ J. Quigley, ${ }^{(6)}$ G. Rong, ${ }^{(1)}$ B. Schmid, ${ }^{(4)}$ J. Schultz, ${ }^{(4)}$ J. T. Shank, (2) Y. Y. Shao, (1) D. L. Shen, ${ }^{(1)}$ H. Y. Sheng, (1) H. Z. Shi, (1) A. Smith, ${ }^{(4)}$ E. Soderstrom, ${ }^{(7)}$ X. F. Song, ${ }^{(1)}$ D. P. Stoker, ${ }^{(4)}$ H. S. Sun, ${ }^{(1)}$ J. Synodinos, ${ }^{(7)}$ W. H. Toki, ${ }^{(5)}$ G. L. Tong, ${ }^{(1)}$ E. Torrence, ${ }^{(6)}$ L. Z. Wang, (1) M. Wang, (1) P. Wang, (1) P. L. Wang, (1) T. J. Wang, ${ }^{(1)}$ Y. Y. Wang, ${ }^{(1)}$ J. S. Whitaker, ${ }^{(2)}$ R. J. Wilson, ${ }^{(2)}$ W. J. Wisniewski, ${ }^{(8)}$ X. D. Wu, ${ }^{(1)}$ D. M. Xi, ${ }^{(1)}$ X. M. Xia, ${ }^{(1)}$ P. P. Xie, ${ }^{(1)}$ X. X. Xie, ${ }^{(1)}$ R. S. $\mathrm{Xu},{ }^{(1)}$ Z. Q. Xu, (1) S. T. Xue, (1) R. K. Yamamoto, ${ }^{(6)}$ J. Yan, (1) W. G. Yan, ${ }^{(1)}$ C. M. Yang, ${ }^{(1)}$ C. Y. Yang, ${ }^{(1)}$ H. B. Yao, ${ }^{(1)}$ M. H. Ye, (1) S. Z. Ye, ${ }^{(1)}$ Z. Q. Yu, (1) B. Y. Zhang, ${ }^{(1)}$ C. C. Zhang, ${ }^{(1)}$ D. H. Zhang, (1) H. L. Zhang, ${ }^{(1)}$ H. Y. Zhang, (1) J. W. Zhang, (1) L. S. Zhang, (1) S. Q. Zhang, (1) Y. Zhang, (1) D. X. Zhao, ${ }^{(1)}$ M. Zhao, ${ }^{(1)}$ P. D. Zhao, (1) W. R. Zhao, (1) J. P. Zheng, (1) L. S. Zheng, (1) Z. P. Zheng, ${ }^{(1)}$ G. P. Zhou, ${ }^{(1)}$ H. S. Zhou, ${ }^{(1)}$ L. Zhou, ${ }^{(1)}$ L. Zhou, ${ }^{(1)}$ X. F. Zhou, ${ }^{(1)}$ Y. H. Zhou, ${ }^{(1)}$ Q. M. Zhu, ${ }^{(1)}$ Y. C. Zhu, ${ }^{(1)}$ Y. S. Zhu, (1) and G. Zioulas ${ }^{(4)}$

\section{(BES Collaboration)}

\author{
${ }^{(1)}$ Institute of High Energy Physics, Beijing 100039, People's Republic of China \\ ${ }^{(2)}$ Boston Unicersity, Boston, Massachusetts 02215 \\ ${ }^{(3)}$ California Institute of Technology, Pasadena, California 91125 \\ (4) University of California, Irvine, California 92717 \\ ${ }^{(5)}$ Colorado State University, Fort Collins, Colorado 80523 \\ (6) Massachusetts Institute of Technology, Cambridge, Massachusetts 02139 \\ ${ }^{(7)}$ Stanford Linear Accelerator Center, Stanford University, Stanford, California 94309 \\ ${ }^{(8)}$ Superconducting Super Collider Laboratory, Dallas, Texas 75237-3946

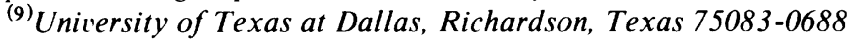 \\ ${ }^{(10)}$ University of Washington, Seattle, Washington 98195 \\ (Received 7 August 1992)
}

\begin{abstract}
The mass of the $\tau$ lepton has been measured at the Beijing Electron-Positron Collider using the Beijing Spectrometer. A search near threshold for $e^{+} e^{-} \rightarrow \tau^{+} \tau^{-}$was performed. Candidate events were identified by requiring that one $\tau$ decay via $\tau \rightarrow e v \bar{v}$, and the other via $\tau \rightarrow \mu v \bar{v}$. The mass value, obtained from a fit to the energy dependence of the $\tau^{+} \tau^{-}$cross section, is $m_{\tau}=1776.9_{-0.5}^{+0.4} \pm 0.2 \mathrm{MeV}$.
\end{abstract}

PACS numbers: $14.60 . \mathrm{Jj}, 13.10 .+\mathrm{q}$

For a conventional charged lepton $l$, the electronic branching ratio $B \mathcal{l}$, lifetime $\tau_{l}$, mass $m_{l}$, and weak coupling constant $G_{l \rightarrow e v \bar{v}}$ are related by

$$
\frac{B l}{\tau_{l}}=\frac{G_{l \rightarrow e v \bar{v}}^{2}}{192 \pi^{3}} m_{l}^{5},
$$

up to small radiative and electroweak corrections. Equation (1) then implies the following relationship among the above parameters for the $\tau$ and $\mu$ leptons:

$$
\left(\frac{G_{\tau \rightarrow e v \bar{v}}}{G_{\mu \rightarrow e v \bar{v}}}\right)^{2}=\left(\frac{m_{\mu}}{m_{\tau}}\right)^{5} \frac{B_{\tau}^{e}}{B_{\mu}^{e}} \frac{\tau_{\mu}}{\tau_{\tau}} .
$$

Particle Data Group (PDG) [1] averages for the above quantities yield $\left(G_{\tau \rightarrow e v \bar{\nu}} / G_{\mu \rightarrow e v \bar{v}}\right)^{2}=0.941 \pm 0.025$, implying a 2.4 standard deviation disagreement with lepton universality [2]. Note that the $\tau$ mass enters to the fifth power in this test of lepton universality.

A measurement of the $\tau^{+} \tau^{-}$production cross section in the region most sensitive to the $\tau$ mass-a few $\mathrm{MeV}$ around threshold-provides the opportunity to measure the $\tau$ mass with greatly improved precision. This paper presents such a measurement made using the Beijing Spectrometer (BES) at the Beijing Electron-Positron Collider (BEPC). The $\tau^{+} \tau^{-}$events are identified by 
means of the $e \mu$ topology, which provides the best combination of high detection efficiency and low background; the mass value is obtained from a fit to the energy dependence of the cross section. The measurement is independent of the $v_{\tau}$ mass.

The BEPC [3] operates in the 3 to $5 \mathrm{GeV}$ center-ofmass energy range. Near $\tau^{+} \tau^{-}$threshold, the peak luminosity is $5 \times 10^{30} \mathrm{~cm}^{-2} \mathrm{~s}^{-1}$, the luminosity-weighted uncertainty in the mean center-of-mass energy is 0.10 $\mathrm{MeV}$, and the spread in the center-of-mass energy of the collider is $\approx 1.4 \mathrm{MeV}$. The absolute energy scale and energy spread are determined by interpolation between the results of repeated scans of the $J / \psi$ and $\psi(2 S)$ resonances.

The BES is a solenoidal detector [3] with a 0.4-T magnetic field. Charged track reconstruction is performed by means of a cylindrical drift chamber which provides solid angle coverage of $85 \%$ of $4 \pi$. The momentum resolution is $\sigma_{p} / p=0.021\left(1+p^{2}\right)^{1 / 2}(p$ in $\mathrm{GeV} / c)$. Measurements of $d E / d x$ with resolution $8.5 \%$ allow particle identification. An inner drift chamber is used for trigger purposes. Scintillation counters measure the time of flight of charged particles over $76 \%$ of $4 \pi$ with a Bhabha resolution of $330 \mathrm{ps}$. A cylindrical 12-radiation-length $\mathrm{Pb} /$ gas electromagnetic calorimeter operating in limited streamer mode covering $80 \%$ of $4 \pi$ achieves energy resolution $\sigma_{E} / E=0.25 / \sqrt{E(\mathrm{GeV})}$, and spatial resolution $\sigma_{\phi}=4.5$ $\mathrm{mrad}, \sigma_{z}=2 \mathrm{~cm}$. End-cap time-of-flight counters and shower counters are not used in this analysis. Finally, a three-layer iron flux return instrumented for muon identification yields spatial resolutions $\sigma_{z}=5 \mathrm{~cm}, \sigma_{r \phi}=3 \mathrm{~cm}$ over $68 \%$ of $4 \pi$ for muons with momentum greater than $550 \mathrm{MeV} / c$.

In the data analysis, the event selection for $e \mu$ candidates requires the following: (1) exactly two oppositely charged tracks having momentum between $350 \mathrm{MeV} / \mathrm{c}$ and the maximum for an electron from $\tau$ decay; (2) each track's point of closest approach to the intersection point to satisfy $|x|<1.5 \mathrm{~cm},|y|<1.5 \mathrm{~cm}$, and $|z|<15 \mathrm{~cm}$; (3) $2.5^{\circ}<\theta_{\text {acol }}<177.5^{\circ}, \theta_{\text {acop }}>10^{\circ}$ (see Ref. [4]), and $\theta_{\text {acol }}+\theta_{\text {acop }}>50^{\circ}$; (4) no isolated photons [5]; (5) one track well identified as a muon in the muon counter, with calorimeter energy $<500 \mathrm{MeV}$, and the other track well identified as an electron using a combination of calorimeter, $d E / d x$, and time-of-flight information.

Monte Carlo simulations yield a detection efficiency of $\approx 14 \%$ for these selection criteria, independent of energy in the threshold region. The background is estimated by applying the same requirements to $5 \times 10^{6}$ events from a data sample taken at the $J / \psi$ energy; seven events meet these criteria, corresponding to a background of 0.12 event in the entire $\tau^{+} \tau^{-}$sample.

The likelihood function used to estimate the $\tau$ mass incorporates the $\tau^{+} \tau^{-}$cross section near threshold. Including the center-of-mass energy spread $\Delta$, initial state radiation [6] $F(x, W)$, and vacuum polarization corrections [7] $\Pi(W)$, the cross section is

$$
\sigma\left(W, m_{\tau}\right)=\frac{1}{\sqrt{2 \pi} \Delta} \int_{0}^{\infty} d W^{\prime} e^{-\left(W-W^{\prime}\right)^{2} / 2 \Delta^{2}} \int_{0}^{1-4 m_{\tau}^{2} / W^{\prime 2}} d x F\left(x, W^{\prime}\right) \sigma_{1}\left(W^{\prime} \sqrt{1-x}, m_{\tau}\right)
$$

where $\sigma_{1}$ is

$$
\sigma_{1}\left(W, m_{\tau}\right)=\frac{4 \pi \alpha^{2}}{3 W^{2}} \frac{\beta\left(3-\beta^{2}\right)}{2} \frac{F_{c}(\beta) F_{r}(\beta)}{[1-\Pi(W)]^{2}}
$$

$W$ is the center-of-mass energy, and $\beta=\left[1-\left(2 m_{\tau}\right]\right.$ $\left.W)^{2}\right]^{1 / 2}$. The Coulomb interaction and final-state radiation corrections are described [8] by the functions $F_{c}(\beta)$ and $F_{r}(\beta)$.

The likelihood function is a product of Poisson distributions, one for each center-of-mass energy. At each point, the number of expected $e \mu$ events $\langle N\rangle$ is given by

$$
\langle N\rangle=\left[\epsilon B \sigma\left(W, m_{\tau}\right)+\sigma_{B}\right] \mathcal{L}
$$

Here, $\epsilon$ is the detection efficiency, $B$ is the product branching fraction for $\tau^{+} \tau^{-}$to $e \mu, \mathcal{L}$ is the integrated luminosity, and $\sigma_{B}$ is the effective background cross section estimated from the $J / \psi$ data sample $\left(\sigma_{B}=0.024 \mathrm{pb}\right)$.

Since the range of center-of-mass energies where the $\tau^{+} \tau^{-}$cross section is most sensitive to the $\tau$ mass is of the order of the beam energy spread around $\tau^{+} \tau^{-}$ threshold, it is important to devise a running strategy to maximize the integrated luminosity in this region. The beam energy is set initially assuming the world average for the $\tau$ mass; in this case, the PDG value is 1784.1 $\mathrm{MeV}$ [1]. Then, after each $250-400 \mathrm{nb}^{-1}$ of integrated luminosity, a new estimate of the mass is made using all the data accumulated to that point; in this way, a new prediction of the most sensitive energy at which to run is obtained. The energy is changed to this new value if the difference is more than the BEPC step size $(\approx 0.4 \mathrm{MeV})$. Following this strategy, an integrated luminosity of $\approx 4.3 \mathrm{pb}^{-1}$ has been accumulated at ten energies within a range of $24 \mathrm{MeV}$. It has been verified by Monte Carlo simulation that this data-driven search strategy provides an unbiased measurement.

The sequence of energies is shown in Fig. 1; the corresponding data are summarized in Table I [9]. The tenstep search yielded seven $e \mu$ events. The eleventh and twelfth points in Table I, taken well above threshold where the cross section varies slowly with energy, provide an improved estimate of the absolute $\tau^{+} \tau^{-}$cross section.

In order to account for uncertainties in the efficiency $\epsilon$, the branching fraction product, and the luminosity, $\epsilon$ is treated as a free parameter in a two-dimensional maximum-likelihood fit for $m_{\tau}$ and $\epsilon$ to the data of Table I. 


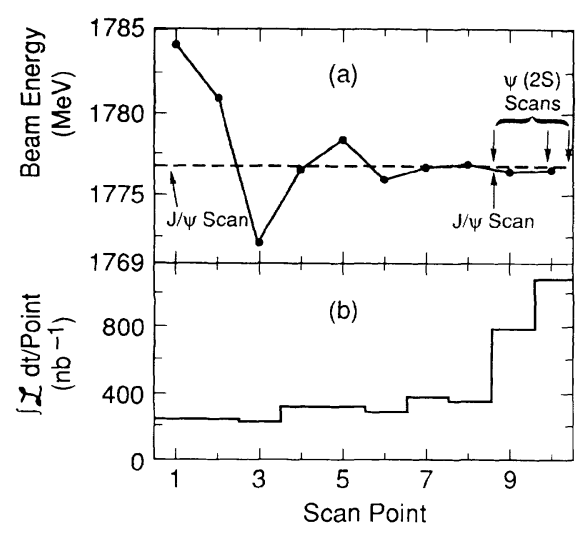

FIG. 1. (a) The convergence of the predicted mass with each consecutive scan point. (b) The integrated luminosity accumulated at each point.

The estimates obtained are $m_{\tau}=1776.9 \mathrm{MeV}$ and $\epsilon=14.1 \%$. The uncertainty in $\epsilon$ is equivalent to the uncertainty in the absolute normalization, and is treated as a source of systematic error. The statistical error [10] in $m_{\tau}, \pm 0.4 \mathrm{MeV}$, is determined from the one-parameter likelihood function with $\epsilon$ fixed to $14.1 \%$ (Fig. 2). The efficiency-corrected cross-section data as a function of corrected beam energy and the curve which results from the likelihood fit are shown in Fig. 2. The quality of the fit is checked by forming the likelihood ratio $\lambda$, with the result [11] $-2 \ln \lambda=3.6$.

Four independent sources of systematic error are considered: uncertainties in the product $\epsilon B \mathcal{L}$, in the absolute beam energy scale, in the beam energy spread, and in the background.

The systematic uncertainty in $\epsilon B \mathcal{L}$ is determined by fixing $m_{\tau}$ at its best-estimate value and finding the values of $\epsilon$ corresponding to $\pm 1 \sigma$ variations in the likelihood function; these efficiencies are $18.3 \%$ and $10.6 \%$. Fixing the efficiency to each of these values in turn and fitting for $m_{\tau}$ yields changes in the predicted mass of $\Delta m_{\tau}$

TABLE 1. A chronological summary of the $\tau^{+} \tau^{-}$data.

\begin{tabular}{ccccc}
\hline \hline Scan point & $\begin{array}{c}W / 2 \\
(\mathrm{MeV})\end{array}$ & $\begin{array}{c}\Delta \\
(\mathrm{MeV})\end{array}$ & $\begin{array}{c}\mathcal{L} \\
\left(\mathrm{nb}^{-1}\right)\end{array}$ & $\begin{array}{c}N \\
(e \mu \text { events })\end{array}$ \\
\hline 1 & 1784.19 & 1.34 & 245.8 & 2 \\
2 & 1780.99 & 1.33 & 248.9 & 1 \\
3 & 1772.09 & 1.36 & 232.8 & 0 \\
4 & 1776.57 & 1.37 & 323.0 & 0 \\
5 & 1778.49 & 1.44 & 322.5 & 2 \\
6 & 1775.95 & 1.43 & 296.9 & 0 \\
7 & 1776.75 & 1.47 & 384.0 & 0 \\
8 & 1776.98 & 1.47 & 360.8 & 1 \\
9 & 1776.45 & 1.44 & 794.1 & 0 \\
10 & 1776.62 & 1.40 & 1109.1 & 1 \\
11 & 1799.51 & 1.44 & 499.7 & 5 \\
12 & 1789.55 & 1.43 & 250.0 & 2 \\
\hline \hline
\end{tabular}
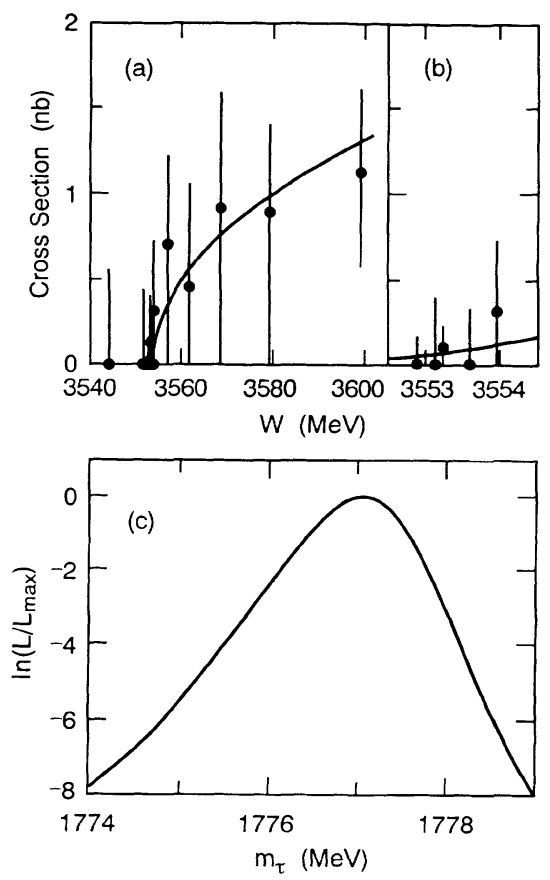

FIG. 2. (a) The center-of-mass energy dependence of the $\tau^{+} \tau^{-}$cross section resulting from the likelihood fit (curve), compared to the efficiency-corrected data. The error bar on each data point is computed by integrating the Poisson likelihood function to obtain the interval containing $68 \%$ of the area. It should be emphasized that the curve does not result from a direct fit to these data points. (b) An expanded version of (a), in the immediate vicinity of $\tau^{+} \tau^{-}$threshold. (c) The dependence of the logarithm of the likelihood function on $m_{\tau}$, with efficiency fixed at $14.1 \%$.

\section{$= \pm{ }_{-0.20}^{0.16} \mathrm{MeV}$.}

The energy scale is determined from several scans of the $J / \psi$ and $\psi(2 S)$ performed during the search (see Fig. 1). The reproducibility of the fits to these scans, together with the other uncertainties listed in Table II, yields a systematic uncertainty [12] of $\Delta m_{\tau}= \pm 0.09 \mathrm{MeV}$.

Fits to the two resonances were also used to measure the beam energy spread and its variation with center-ofmass energy and beam current. The uncertainty in center-of-mass energy spread is $\pm 0.08 \mathrm{MeV}$, yielding a systematic error $\Delta m_{\tau}= \pm 0.02 \mathrm{MeV}$.

TABLE II. Contributions to the uncertainty in the energy scale.

\begin{tabular}{lr}
\hline \multicolumn{1}{c}{ Quantity } & \multicolumn{1}{c}{$\begin{array}{c}\text { Error } \\
(\mathrm{MeV})\end{array}$} \\
\hline$W_{M}:$ BEPC measured center-of-mass energy & $\delta W_{M}=0.10$ \\
$M_{\psi}:$ BEPC value for $J / \psi$ mass & $\delta M_{\psi}=0.18$ \\
$M_{\psi}:$ BEPC value of $\psi(2 S)$ mass & $\delta M_{\psi^{\prime}}=0.15$ \\
$T_{\psi}:$ PDG value for $J / \psi$ mass ${ }^{\mathrm{a}}$ & $\delta T_{\psi}=0.09$ \\
$T_{\psi}:$ PDG value for $\psi(2 S)$ mass $^{\mathrm{a}}$ & $\delta T_{\psi}=0.10$ \\
\hline \hline
\end{tabular}

${ }^{a}$ Reference [1]. 


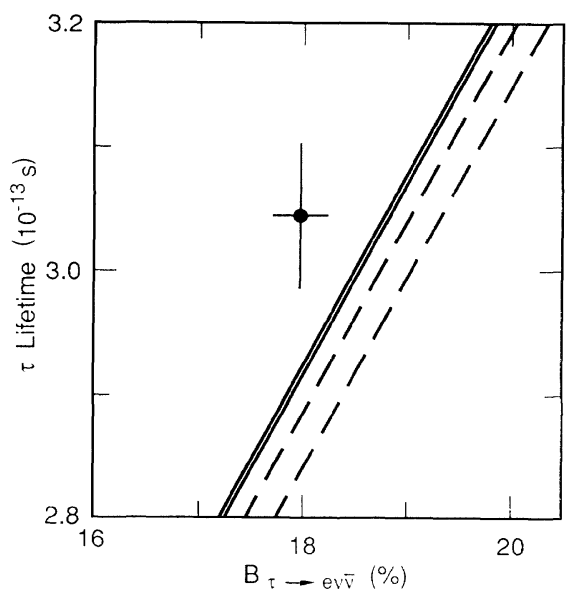

FIG. 3. The variation of $\tau_{\tau}$ with $B_{\tau}^{e}$, given by Eq. (1) under the assumption of lepton universality; the $\pm 1 \sigma$ bands obtained using $m_{\mathrm{\tau}}$ from this experiment (solid lines) and using the PDG value (dashed lines) are shown in comparison to the point corresponding to the PDG values ( $1 \sigma$ error bars).

Finally, the systematic error due to uncertainty in the background is estimated from the $1 \sigma$ Poisson errors on the seven $J / \psi$ background events and the uncertainty in the hadronic cross section at $\tau^{+} \tau^{-}$threshold. The resulting uncertainty is $\Delta m_{\tau}= \pm 0.01 \mathrm{MeV}$.

These independent systematic errors are added in quadrature to yield a total systematic error of $\Delta m_{\tau}= \pm 0.18$ MeV.

In conclusion, using a maximum-likelihood fit to $\tau^{+} \tau^{-}$cross-section data near threshold, the mass of the $\tau$ lepton has been measured as $m_{\tau}=1776.9 \pm_{0.5}^{+0.4} \pm 0.2$ $\mathrm{MeV}$, where the first error is statistical and the second systematic. This result is $7.2 \mathrm{MeV}$ below the PDG average [1] $(1784.1 \pm 2.7 \mathrm{MeV})$ and has significantly smaller errors [13]. Inserting this new value in Eq. (2), the coupling strength ratio becomes

$$
\left(G_{\tau \rightarrow e v \bar{v}} / G_{\mu \rightarrow e v \bar{v}}\right)^{2}=0.960 \pm 0.024,
$$

so that the deviation from lepton universality is reduced from 2.4 to 1.7 standard deviations (see Fig. 3). It should be noted also that this new result for $m_{\tau}$ yields a reduction in the upper limit on $m_{v_{\tau}}$ (see Ref. [13]).

We would like to thank the BEPC staff and the Computer Center at IHEP for their outstanding efforts, and also Y. S. Tsai and J. M. Wu for helpful discussions about the $\tau^{+} \tau^{-}$cross section near threshold. This work was supported in part by the National Natural Science Foundation of China under Contract No. 19290400 (IHEP); by the Department of Energy under Contracts
No. DE-AC03-76SF00515 (SLAC), No. DE-FG0392ER40701 (Caltech), No. DE-FG03-91ER40679 (UC Irvine), No. DE-FG02-91ER40676 (Boston University), No. DE-AC02-76ER03069 (MIT), No. DE-AC 3589ER40486 (SSC), and No. DE-FG05-92ER40736 (UT Dallas); by the Texas National Research Laboratory Commission (Rocky Mountain Consortium for High Energy Physics) under Contracts No. RGFY91B5 and No. RGFY92B5 (Colorado State); and by the National Science Foundation (University of Washington).

[1] Particle Data Group, K. Hikasa et al., Phys. Rev. D 45, S1 (1992).

[2] W. J. Marciano, Phys. Rev. D 45, 721 (1992). The measurements quoted in this reference have been superseded by Ref. [1].

[3] M. H. Ye and Z. P. Zheng, in Proceedings of the 1989 International Symposium on Lepton and Photon Interactions at High Energies (Stanford University, Stanford, 1989), p. 122.

[4] Acoplanarity, $\theta_{\text {acop }}$, is defined as the angle between the planes spanned by the beam direction and the momentum vector of $e$ and $\mu$, respectively. Acollinearity, $\theta_{\text {acol, }}$ is defined as the angle between the momentum vectors of $e$ and $\mu$.

[5] An isolated photon has an energy $>60 \mathrm{MeV}$ and is separated from the nearest charged track by $>12^{\circ}$.

[6] É. A. Kuraev and V. S. Fadin, Yad. Fiz. 41, 733 (1985) [Sov. J. Nucl. Phys. 41, 466 (1985)].

[7] F. A. Berends and G. J. Komen, Phys. Lett. 63B, 432 (1976).

[8] M. B. Voloshin, Report No. TPI-MINN-89-33-T, 1989 (unpublished).

[9] $\Delta$ was determined according to the equation $\Delta$ $=(A \bar{I}+B)\left(C W^{2}+D\right)$, where $\bar{I}$ is the average beam current. $A, B, C$, and $D$ are fitted to beam current measurements and to measurements of the energy spreads at the $J / \psi$ and $\psi(2 S)$ resonances.

[10] It has been verified by simulation that this uncertainty corresponds to a $68 \%$ confidence interval.

[11] In the large statistics limit, $-2 \ln \lambda$ would obey a $\chi^{2}$ distribution for 10 degrees of freedom.

[12] Assuming a linear relation between measured energy $W_{M}$, and the corrected value $W$, the latter is given by $W$ $=T_{\psi}+\left(W_{M}-M_{\psi}\right)\left[\left(T_{\psi^{\prime}}-T_{\psi}\right) /\left(M_{\psi^{\prime}}-M_{\psi}\right)\right]$ in the notation of Table II. At $\tau^{+} \tau^{-}$threshold the resulting mass scale correction is $W-W_{M}=-0.74 \mathrm{MeV}$, with corresponding uncertainty $\delta W=0.18 \mathrm{MeV}$.

[13] The ARGUS Collaboration has also reported [H. Albrecht et al., Report No. DESY 92-086, 1992 (to be published)] a new measurement of the $\tau$ mass, $m_{\tau}$ $=1776.3 \pm 2.4 \pm 1.4 \mathrm{MeV}$. 